\section{Vidéosurveillance à Johannesburg Impacts sur la population et le territoire}

\section{Lucas Oesch, Genève}

\section{Introduction}

Il est devenu commun, lorsqu'on se promène dans une ville, de croiser le regard d'une caméra suspendue dans l'espace public. Johannesburg, que beaucoup présentent comme une, voire la «ville du crime», ne fait pas exception, puisqu'un gigantesque système de vidéosurveillance a été installé dans son Central Business District (CBD). Ancien centre névralgique de la ville, une imagerie négative lui est désormais associée du fait de la criminalité et du délabrement. Ces perceptions oscillent entre mythes et réalité. Loin d'être l'espace à l'abandon ni le coupe-gorge si souvent décrit, le CBD est encore le lieu d'une intense activité économique et près de $800^{\prime} 000$ pendulaires s'y rendent quotidiennement. Il est également le lieu d'une certaine diversité sociale, économique et «raciale». Pour Guillaume (1997: 31), toutefois, il «semble abusif de parler d'intégration» de cette diversité; il est plutôt question de «collision».

Dans ce contexte de ville post-apartheid, les autorités et les acteurs actifs dans le développement urbain ont l'ambition de faire de «Joburg» une «world-class city» et sont décidés à «s'attaquer» au re-développement du CBD afin de lui redonner son aura d'antan. C'est dans le cadre de cette reconstruction que l'utilisation de caméras de surveillance a été adopté afin de résoudre le problème de la criminalité.

Cet article commencera par la présentation de quelques éléments théoriques concernant la vidéosurveillance et sa relation au territoire et à la population. Une description du système de vidéosurveillance du CBD de Johannesburg sera ensuite effectuée et soulignera que la surveillance opérée se concentre principalement sur les «lieux de pouvoir». L'article se poursuivra ensuite par une analyse des impacts des caméras sur le phénomène criminel. Il sera fait mention du caractère incertain de ces effets. Enfin, il sera question des effets spatiaux et sociaux de la vidéosurveillance, et au final de son impact sur la diversité sociale, économique et «raciale» du CBD. Outre le développement d'une hiérarchisation des espaces et d'un nouveau processus de territorialisation, l'article mettra en évidence l'instauration d'une culture publique privatisée, qui semble exclure toute une frange de la population.

\section{La vidéosurveillance dans la ville: un processus de territorialisation}

La littérature sur la vidéosurveillance est principalement anglo-saxonne (Fyfe \& BANNISTER 1996, 1998; Norris \& Armstrong 1998) même si d'intéressantes contributions francophones existent également (BÉTIN Martinais \& Renard 2003; November, Klauser \& Ruegg 2002). Selon Bannister, Fyfe \& KeARns (1998: 22), la vidéosurveillance dans l'espace urbain est développée en fonction du postulat suivant lequel

«CCTV [Closed Circuit Television] combats crime leading to improved levels of safety, and (...) helps ameliorate the fear of crime contributing to a 〈feel-good factor».

Ces auteurs considèrent également que

«CCTV has been promoted as a valuable tool in the drive to revive the economic fortunes of the city centre. Through reducing crime and the fear of crime it is hoped that the city centre will become a more attractive place for business to locate and the consumer to shop» (ibid.).

Les débats ayant cours au sein du monde politique concernant la vidéosurveillance se focalisent souvent sur les questions relatives à la liberté individuelle ou à la vie privée. Cependant, il est nécessaire de dépasser ces considérations, comme l'explique Lyon (2001: 53), «safety, security and social order are all seen by most people as positive accomplishment. (...) [However] the question is, what other effects accompany the positive face of surveillance, especially as it is automated and informatized? And, are the effects of surveillance positive for all or just for some?»

Cet article se concentre sur ces «autres effets», c'est-àdire qu'il analyse les impacts spatiaux et sociaux de la vidéosurveillance et non les questions d'atteinte éventuelle à la vie privée des individus. RAFFESTIN (1997) a expliqué comment les pratiques scientifiques et techniques participent à un processus de territorialisation. L'adoption de médiateurs scientifiques et techniques (en l'occurrence la vidéosurveillance comme nouvelle technique de surveillance), dans le cadre d'un processus de «domestication» ou de «simulation» de l'espace, conduit à une nouvelle territorialisation de celui-ci. Comme l'affirment November, KLauser \& Ruegg (2002: 161), «l'espace surveillé change, ses qualités se transforment». Par ailleurs, RAFFEstin (1997: 94) remarque que

«les formes sociales constituent [notamment] la 〈matière〉 sur laquelle les processus de la culture - scientifiques et techniques entre autres - ne laissent pas de s'exercer». 
Dans la suite de l'article, nous allons donc observer le processus de territorialisation que la vidéosurveillance génère à Johannesburg, et ses impacts sur les formes spatiales et sociales.

\section{La vidéosurveillance dans le CBD: une défense des «lieux de pouvoir»}

Dans cette partie, nous allons décrire le système de vidéosurveillance de l'espace public du CBD de Johannesburg, avant d'analyser, dans la partie suivante, ses répercussions sur le territoire et la population. Selon BREMNER (1998: 58), à Johannesburg,

«the major institutions, seeing their investments eroded by capital flight and demographic shifts, have pointed a finger at the new government and its inability to deal with crime or manage the public environment. This argument has been adopted by those in public office (...) and they have launched many fire-fighting initiatives to combat crime, including (...) the creation of $<$ Business Improvement District>, and the installation of closed-circuit television cameras».

L'entreprise Business Against Crime Surveillance Technology (BACST), une filiale de Business Against Crime South Africa (BAC), s'est vu confier la mission de développer un système de vidéosurveillance dans le CBD. Sur son site web, BAC mentionne qu'elle est une compagnie privée créée en 1996 dont le but est «to make South Africa a safer place to live, work and do business » (Business Against Crime South Africa (BAC) 2003). Entre 2003 et 2004, BACST a pris le nom de Cueincident. Selon un responsable de la compagnie interrogé, ce changement de nom a été effectué afin de souligner que la compagnie utilise dorénavant son système de surveillance également à d'autres fins que la lutte contre le crime, telles que la surveillance de la circulation routière par exemple. Lorsqu'on se renseigne sur les raisons de l'installation du système dans le CBD, le même responsable répond que:

«We identified with the South African Police the hotspots, the bad areas in the centre city. (...) It is also economically driven, because the major businesses operate in the CBD. Big business does not operate in Hillbrow. Hillbrow is a residential component. So the business people were becoming more and more anxious about the security of their personnel, and they couldn't get clients to come into the city to deal with them because they were afraid of crime, so that was why we started in the city [centre]» (Cueincident Surveillance Technology, Johannesburg, Marketing and Communication Division, 24 mars 2004).

La protection de la clientèle des agents économiques opérant dans le CBD a donc été la motivation du développement du système de vidéosurveillance dans le centre-ville. Les quartiers centraux à vocation rési- dentielle, comme Hillbrow, ont été exclus des zones à surveiller. L'installation a débuté en 2000 avec un projet comprenant une douzaine de caméras. En 2004 le système en comptait près de 200 réparties dans le CBD. Le coût du système n'est pas connu avec précision. D'après un magazine économique, il avoisine les 157 millions de Rands sud-africains [environ 16 millions d'Euros], bien qu'il ne soit pas possible de savoir s'il s'agit du coût d'installation ou s'il comprend les frais d'entretien et de fonctionnement (CIARAn 2003). Par ailleurs, un responsable de la compagnie explique que plus de la moitié des coûts est payée par la municipalité; le reste étant à la charge d'entités privées qui bénéficient de la surveillance des caméras - dépôt ferroviaire et banques notamment (CuEINCIDENT Surveillance Technology, Johannesburg, Marketing and Communication Division, 24 mars 2004 ).

Le système de surveillance de Cueincident fonctionne uniquement avec ses propres caméras et n'utilise pas d'autres caméras possédées par des particuliers. Il s'agit de ce que November, Klauser \& Ruegg (2002: 159) appellent une surveillance de «surface», qui s'oppose à celle de «points isolés» dans l'espace. Les caméras sont munies de zooms puissants et peuvent effectuer des mouvements rotatifs (Fig. 1). Elles sont installées au niveau des trois premiers étages des immeubles ainsi que sur leur toit. Le système est centralisé, c'est-à-dire que toutes les images des caméras sont diffusées dans une unique salle de contrôle située dans le Carlton Centre, divisée en blocs (appelés «consoles») composés de 16 écrans chacun. Chaque écran diffuse l'image d'une seule caméra en continu. Deux employés de Cueincident visionnent en permanence les images d'un bloc. L'observateur regarde les 16 écrans, alors que l'opérateur, qui possède un dix-septième écran, se concentre, selon les recommandations faites par l'observateur, sur l'image d'une caméra en particulier qu'il peut contrôler manuellement à distance. Il y a également une salle de supervision où des agents du South African Police Service (SAPS) du Johannesburg Metropolitan Police Department (JMPD) et, occasionnellement, des employés du Central Johannesburg Partnership (CJP), observent les images sélectionnées par l'opérateur et ont la possibilité d'informer leurs collègues qui patrouillent en ville. Cueincident mentionne un temps de 60 secondes entre le moment de l'observation d'un «incident» et celui d'une intervention.

Le système de Cueincident comporte à la fois les caractéristiques d'une surveillance de prévention, mais également de répression, selon les catégories établies par Vitalis (1998). La présence des caméras dans l'espace public est censée dissuader l'individu de commettre un crime, mais une réponse rapide est également prévue en cas «d'incidents» se produisant, ou sur 


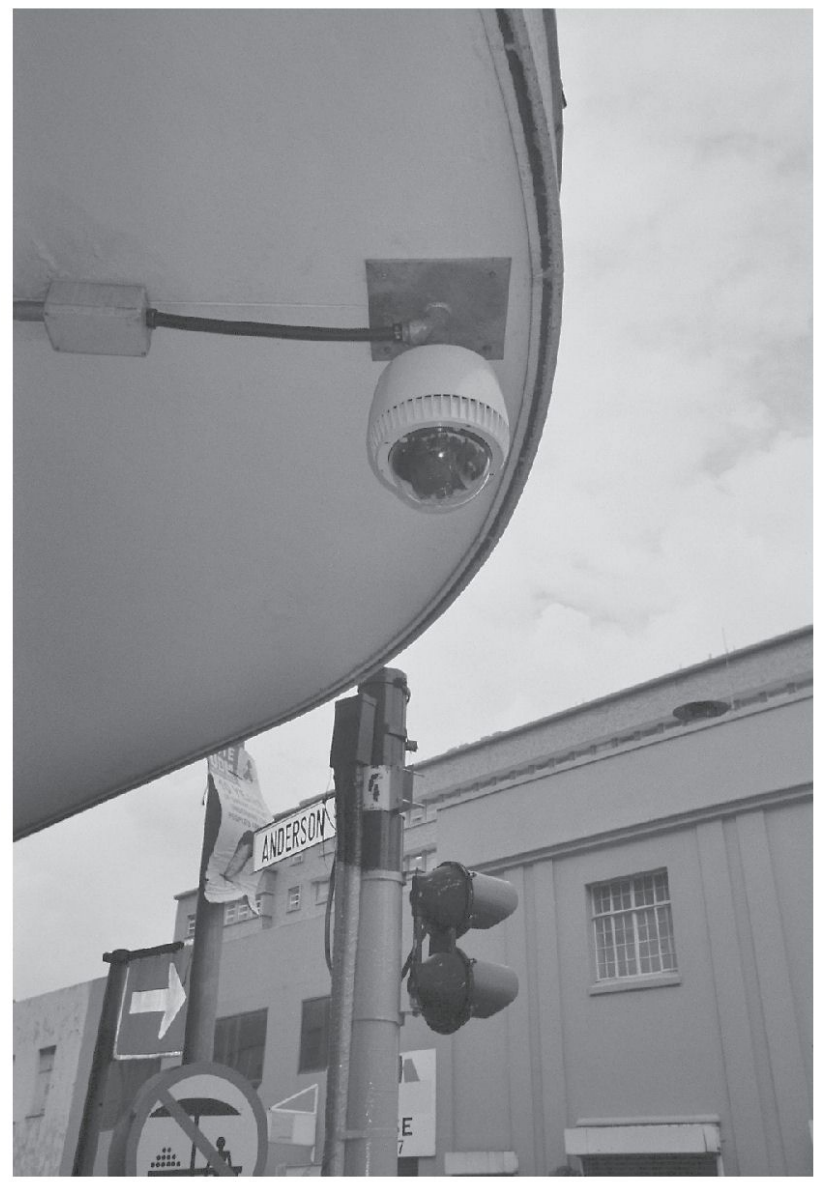

Fig. 1: Une caméra de vidéosurveillance dans le CBD Eine Videoüberwachungskamera im $C B D$ A CCTV camera in the CBD

Photo: L. OEsch 2004

le point de se produire. Sur son site web, Cueincident (2004) mentionne qu'elle effectue une surveillance de type «proactif»:

«This means observing people carefully, reading their body language. (...) Suspicious people are further observed, and if a crime is considered imminent, we ensure that ground response is at hand, to ask them what they are about, and let them know that they are being watched. This way, crimes are stopped before they happen».

Bétin, Martinais \& Renard (2003: 17) remarquent dans leur étude de la vidéosurveillance en ville de Lyon, qu'à travers ce procédé, «le préventif se décline sur l'anticipatif et le répressif»; ce qui ne reste pas sans conséquences sur la diversité sociale, comme la prochaine partie le montrera. Il existe une liste des «incidents» sur lesquels les employés de Cueincident doivent se focaliser, cependant, il demeure que l'observateur et l'opérateur décident du sujet sur lequel ils veulent se concentrer, d'après leur analyse de ce que Cueincident nomme le «body language» des individus. Comme l'explique un gestionnaire du système interrogé,

«the employees are people who know the streets well and they know when somebody is behaving bizarrely».

Ils se concentrent donc sur «everything that doesn't look normal» (Cueincident Surveillance Technology, Johannesburg, Operations Projects Division, 24 mars 2004).

Les caméras de Cueincident se trouvent principalement dans l'espace public (rues, places, parcs), de même que dans des halls de banques, dans un complexe commercial ou près d'un dépôt ferroviaire. Selon un document publicitaire de la compagnie, le système du CBD une fois complet devrait compter 360 caméras disposées entre la limite du Braamfontein Ridge au nord, Ellis Park à l'est, l'autoroute M2 au sud et l'autoroute M1 à l'ouest (CUEINCIDENT 2003). En 2004 , un gestionnaire du système que nous avons interrogé indiquait qu'il y avait 186 caméras en fonction (Cueincident Surveillance Technology, Johannesburg, Operations Projects Division, 24 mars 2004). Etant donné que leur localisation n'est pas une information publique, nous avons effectué un inventaire des caméras en parcourant les rues du CBD (Fig. 2). Dans notre zone d'observation, nous avons recensé 87 caméras de Cueincident présentes dans l'espace public auxquelles s'ajoutent 5 caméras observées dans le Carlton Centre Mall, ainsi que 33 caméras dont nous avons vu les images dans la salle de contrôle (une située en haut du Carlton Centre, 16 au dépôt ferroviaire de City Deep et 16 dans des halls de banques), soit un total de 125 caméras. Notre inventaire n'est pas complet car il manque 61 caméras sur les 186 que Cueincident prétend avoir installées, bien que seuls 160 écrans aient été répertoriés dans la salle de contrôle. Nous pouvons néanmoins effectuer des observations relatives à la localisation de ces caméras dans le CBD.

Les zones vidéosurveillées dans le CBD recouvrent principalement des espaces symbolisant un pouvoir économique, politique ou culturel (Fig. 2). D'une part, les caméras ont été installées dans le CBD, et non dans un quartier résidentiel central comme Hillbrow, qui a la réputation d'être aussi dangereux que le CBD, mais qui ne possède que peu de «clientèle» économique, politique ou culturelle. D'autre part, la même observation est valable en ce qui concerne la répartition des caméras à l'intérieur du CBD: la surveillance des alentours du Carlton Centre (Fig. 3) et de la banque ABSA (zone 2), de Marshalltown dans le Financial District (zone 5), d'une partie des quartiers de Diagonal Street (zone 6) Government Precinct (zone 4), Gandhi Square (zone 3), et Newtown (zone 8), est une surveillance d'espaces liés à l'activité économique. La surveillance de la partie 


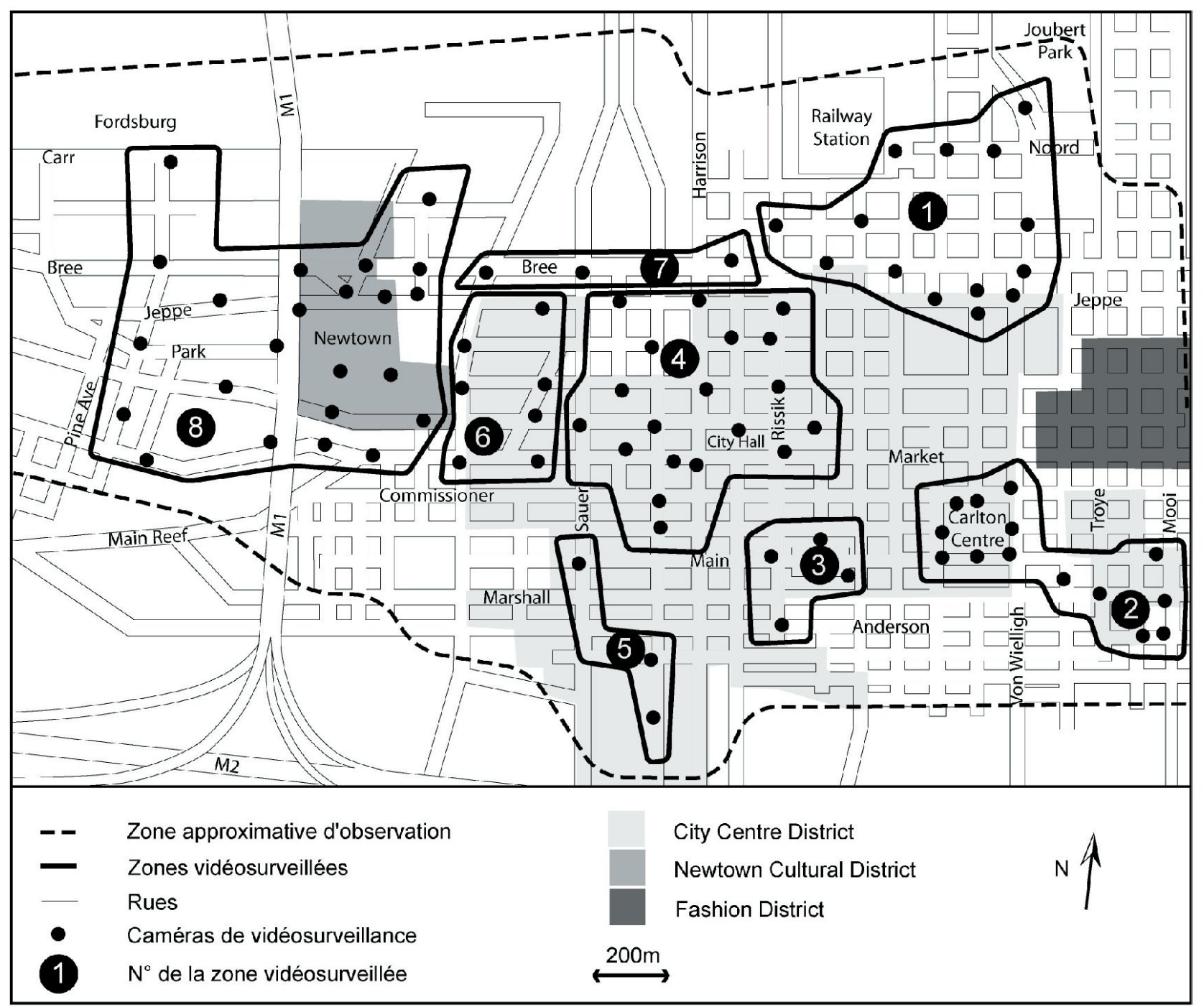

Fig. 2: Localisation des caméras dans le CBD de Johannesburg Lage der Videoüberwachungskameras im $C B D$ von Johannesburg Location of cameras in the CBD of Johannesburg Source du plan des rues: TomLinson 1999; cartography: L. OEsch

restante de Newtown - qui comprend le Newtown Cultural District et le quartier soumis à un processus de gentrification de Fordsburg -, de même que celle de Gandhi Square, représente une surveillance d'espaces d'un (nouveau) pouvoir culturel. Enfin, la surveillance de la partie restante du Government Precinct est une surveillance d'espace à forte connotation politique. A noter également qu'une grande partie de ces espaces est comprise dans le City Centre Business Improvement District (BID). Les caméras semblent donc avoir été installées principalement à l'attention d'une certaine clientèle économique, politique et culturelle, une remarque qui va dans le même sens que celle de Bétin, Martinais \& RENARD (2003:11), lesquels observent à Lyon que «la vidéosurveillance apparaît d'emblée comme l'une des mesures (...) susceptible d'apporter une réponse rapide et visible aux attentes exprimées par une partie de la population et les commerçants du centre-ville en particulier».

Cependant, à Johannesburg, la vidéosurveillance du quartier de Noord Street (zone 1) et de celui de Bree Street (zone 7) représente l'exception à cette défense des «lieux de pouvoir» dans le CBD (Fig.4). Il s'agit là d'une surveillance de la foule dans l'espace public et résidentiel, qui ne concerne cependant que 17 caméras sur les 125 répertoriées. Le fait que ces zones (surtout celle de Noord Street) aient plutôt mauvaise réputation et qu'elles se situent à proximité directe des 


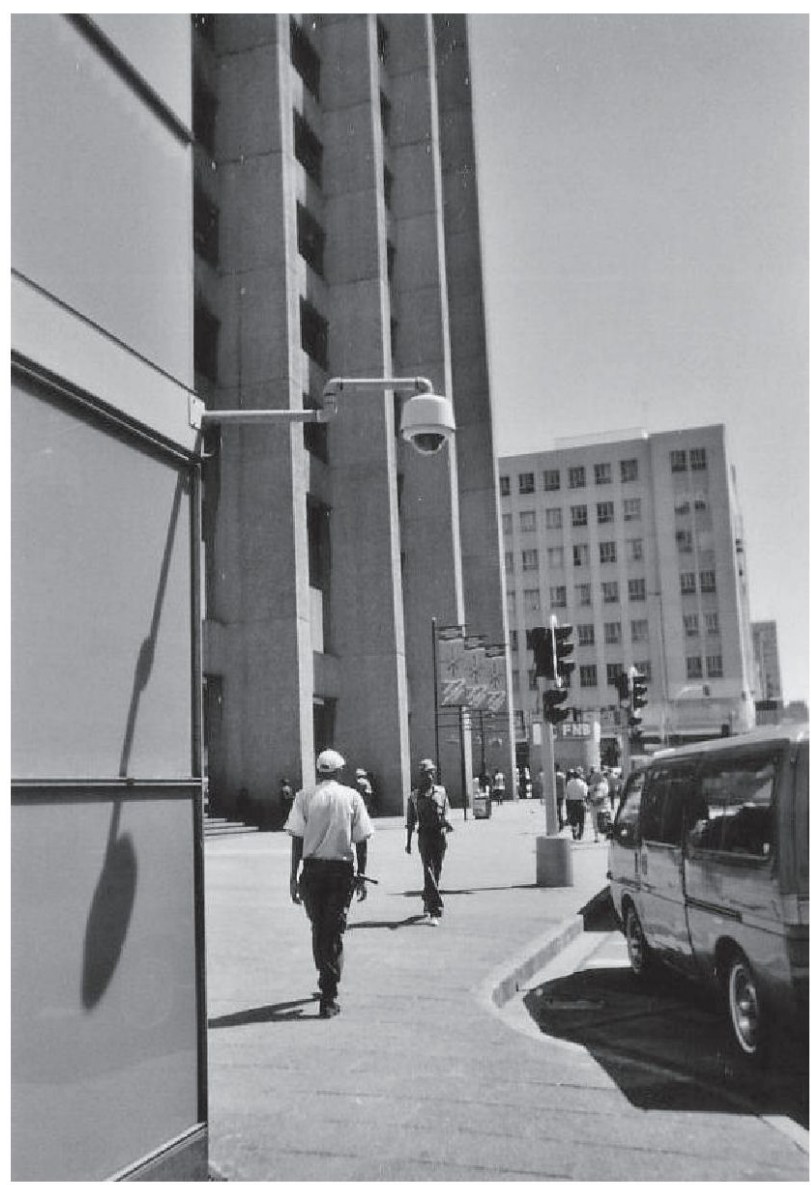

Fig. 3: Une caméra et un garde privé aux abords du Carlton Centre

Eine Kamera und ein privater Wachmann in der unmittelbaren Umgebung des Carlton Centre

A camera and a private security guard near the Carlton Centre

Photo: L. Oesch 2004

lieux de pouvoir explique peut-être l'installation de caméras en leur sein. De plus, la présence de la vidéosurveillance traduit éventuellement un désir de réappropriation de ces deux lieux dans lesquels se situe un certain nombre de bâtiments commerciaux et de bureaux en friche.

\section{Impacts spatiaux et sociaux de la vidéosurveillance}

Après avoir établi que la vidéosurveillance dans le CBD se concentrait dans les lieux de pouvoir, cette partie se donne pour objectif d'évaluer successivement les impacts de ce mode de surveillance sur la criminalité, la population et le territoire, ainsi que sur la culture publique produite dans ce dernier.
Peu après l'installation du système, une diminution de la criminalité a été annoncée. En 2003, BACST revendiquait une diminution de $80 \%$ de la criminalité dans le CBD ( $90 \%$ pour les crimes violents et $75 \%$ pour les autres), alors que la compagnie possédait 184 caméras (Cox 2003). Sur le site web de la municipalité (Greater Johannesburg Metropolitan Council (GJMC) 2004), le directeur de Cueincident mentionne également que

«the kinds of events being recorded now involve petty and largely opportunistic criminal activities».

Cependant, le rapport annuel 2002-2003 du SAPS (South African Police Service (SAPS) 2003) dévoile que la division policière de Central Station qui recouvre le CBD reste parmi les divisions les plus affectées du pays en termes de meurtres et de brigandages («aggravated robberies»). Un commissaire du SAPS interviewé ne semble pas non plus corroborer ces chiffres puisqu'il indique que

«when they say crime has dropped, maybe what they are talking round about, maybe, between $15 \%$ and $20 \%$, not that, what they are talking. Maybe they've got their own stats, but [not] from the police side» (South African Police Service (SAPS), Johannesburg Central Police Station, Crime Prevention Unit, 7 avril 2004).

Une étude réalisée dans la province du Gauteng détaille la façon dont BACST réalise ses statistiques. Un gestionnaire du système y explique que

«BAC works on the basis of incidents observed by the operators. For instance, if operators recorded 100 incidents during the first month of monitoring, and now only records 50 incidents per month, it represents a $50 \%$ reduction in crime. (...) These incidents not only include crimes that have been committed, but also possible crimes that have been prevented from being committed» (PLEssis \& Holtmann 2002: 20).

La diminution de $80 \%$ de la criminalité représente le taux enregistré par la compagnie; or ce taux est calculé sur la seule base d'un décompte mensuel des «incidents observés» par les opérateurs. Selon un chercheur de l'Institute for Security Studies (ISS) à Pretoria, le décalage entre les statistiques de la compagnie qui recense une forte baisse de la criminalité d'une part et le fait que Central Station soit parmi les divisions les plus affectées par les meurtres et les brigandages au regard des statistiques policières d'autre part, peut s'expliquer par un déplacement de la criminalité en dehors du champ des caméras: dans des espaces où le système est absent, sous les avant-toits ou à l'intérieur des bâtiments, de même que derrière les stands des vendeurs de rues ou derrière des arbres (INSTItute for Security Studies (ISS), Pretoria, Crime and Justice Program, 11 mars 2004). Dans le même sens, un commissaire du SAPS indique que le CBD n'est pas «CCTV accommodate» et que de nombreux 


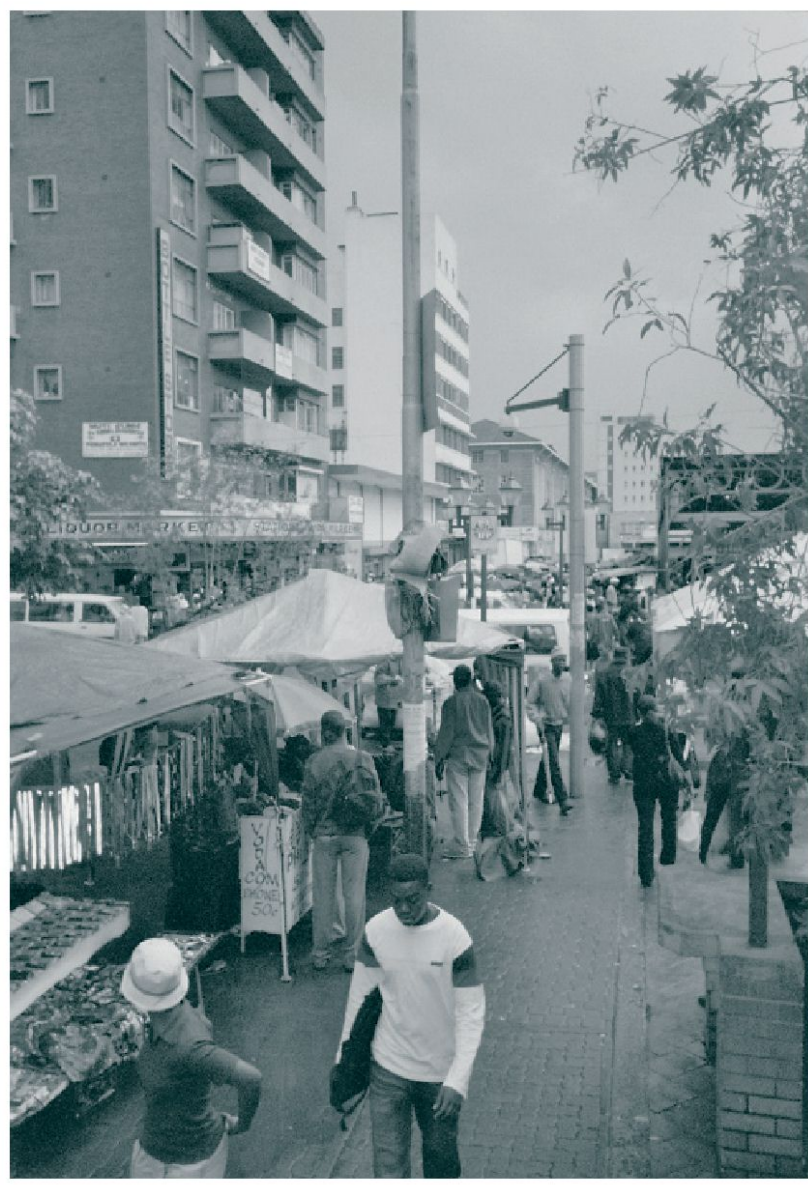

Fig. 4: Une caméra aux environs de Noord Street Eine Kamera in der Umgebung der Noord Street A camera near Noord Street

Photo: L. Oesch 2004

équipements urbains obstruent le regard des caméras. D'après lui, les «criminels» ont aussi développé des nouvelles techniques afin d'esquiver le champ de vision des caméras pendant leurs agissements. Par ailleurs, il signale que le choix des zones vidéosurveillées dans le CBD n'est pas forcément le plus adéquat par rapport à la criminalité:

«the area around Noord (...) that's were most of the crimes are happening, not in, around the banks» (SouTH African Police Service (SAPS), Johannesburg Central Police Station, Crime Prevention Unit, 7 avril 2004).

En définitive, il semble que les effets du système de vidéosurveillance sur la criminalité soient moins évidents qu'il n'y paraît au premier abord. De plus, il est possible de constater qu'il existe un décalage entre Cueincident, d'un côté, qui construit une pratique de surveillance susceptible de lui garantir des résultats et, d'un autre côté, la police qui fonctionne selon des critères différents.
Après avoir discuté des questions liées à l'efficacité de la vidéosurveillance, nous allons maintenant analyser ses impacts sur la population et le territoire. D'une part, nous assistons à un renforcement de la hiérarchisation des espaces, en raison de la répartition inégale des caméras sur le territoire. La vidéosurveillance ajoute une plus-value aux espaces de pouvoir. De ce fait, la vidéosurveillance participe à la

«production 〈d'espaces〉 par exaltation de certains lieux et mise à l'écart d'autres» (RAFFESTIN 1997:96).

D'autre part, nous pouvons observer, comme BÉTIN, Martinais \& Renard (2003: 22), une

«construction de diverses formes de déviance, au sens d'une redéfinition collective des normes sociales qui amènent à les qualifier comme telles».

Comme le signalent Norris \& Armstrong (1998: 8) il est important de tenir compte des schèmes d'interprétation qui guident les jugements des opérateurs dans leur mission de surveillance. A Johannesburg, afin de repérer un éventuel criminel, les employés de Cueincident se focalisent sur l'observation du «body language» des individus. Un reportage d'un magazine économique sud-africain détaille ce procédé:

«This is where Cueincident's operator training and intellectual capital come into play. The body language of someone about to commit a crime is different to someone going about his business: while ordinary people go about their business with purpose, the criminal moves furtively through the crowd, looking around for opportunities. Cueincident operators are trained to look for this kind of behaviour and alert law enforcement officials before a crime is committed» (CIARAN 2003).

Nous avons mentionné précédemment qu'à travers ce procédé le préventif se décline sur l'anticipatif et le répressif, rappelant la nouvelle de science fiction de Dick (2002 [1956]) où le système «precrime» permet de détecter un crime avant qu'il ne se produise et de réprimer le criminel potentiel. BÉTIN, MaRTINAIs \& RENARD (2003: 20) expliquent qu'à travers un tel système anticipatif et répressif,

«ce ne sont pas les actes (non encore commis) qui sont considérés comme déviants, mais les comportements tels qu'ils apparaissent à l'opérateur par l'image».

Les individus «déviants» et à réprimer de façon anticipative deviennent ceux dont les comportements ne correspondent pas aux normes sociales prédéfinis par certains groupes sociaux. Ainsi, la production de territoire à travers la vidéosurveillance correspond à une nouvelle territorialisation qui se fait au détriment d'un processus de «dé-territorialisation» pour certains groupes sociaux. Autrement dit, l'appropriation sélective et la privatisation des espaces publics par certains groupes sociaux, à l'aide de la vidéosurveillance entraînent la répression et l'exclusion d'autres groupes sociaux en fonction de leurs comportements et pas nécessairement parce qu'ils sont des criminels en 
puissance. Fyfe \& BANNister (1998: 261) vont dans le même sens lorsqu'ils mentionnent que l'utilisation de la vidéosurveillance

«includes the exclusion from public space of those unable or unwilling to subscribe to the norms».

Il reste à déterminer, dans le cas de Johannesburg, quels sont les comportements qui sont visés par ces nouvelles normes influençant le fonctionnement de la vidéosurveillance. Une analyse minutieuse du travail des employés de Cueincident serait nécessaire afin d'établir précisément les comportements en question et la mesure dans laquelle ils se trouvent exclus ou réprimés de façon anticipative. A défaut d'une telle information, il est tout de même possible de suggérer certaines pistes.

\section{Piste de recherche: la production d'une nouvelle culture publique}

LÉvy (1994: 331) remarque qu'il existe une «inégalité des agents en compétition dans l'espace central» d'une ville et que «les logiques économiques avantagent les consommateurs solvables». En s'appuyant sur ces considérations, et en se rappelant que le système de vidéosurveillance dans le CBD a pour principal objet la protection de la clientèle des agents économiques, il est possible de postuler que les réprimés ou les exclus sont ceux ne voulant pas ou ne pouvant pas souscrire à ce que CHRISTOPHERSON (1994) appelle la «consumer citizenship».

Afin d'étayer ces propos, il est intéressant d'analyser la nouvelle culture publique produite à travers le processus de territorialisation. DirsuweIt (2002) et Guillaume (2004) expliquent que Johannesburg est caractérisée par une culture de la peur qui s'exprime par une peur de la violence et de la criminalité qui peut être assimilée à une peur de l'autre et de la différence. Dans leur étude portant sur la province du Gauteng, Plessis \& Holtmann (2002: 5) concluent que

«electronic surveillance systems have an undisputed positive impact on people's perceptions of safety».

D'après cette recherche, la vidéosurveillance semble donc être un moyen d'altérer la culture de la peur. Cependant, il est loisible de se poser la question de la culture publique qui y est produite. FYFE \& BANNISTER (1998: 257) considèrent à ce propos que les pratiques de vidéosurveillance accompagnent des procédés de transformation des «downtown[s] as mall[s]». La sécurisation de l'espace est ainsi principalement motivée par ce que ZuKIN (1995: 31) appelle des «visions consommables» de la civilité et de l'espace qui façonnent une nouvelle culture publique basée sur la consommation. Christopherson (1994: 413) résume cela lorsqu'elle dit que l'urbanité est «narrowed and redefined as a consumption experience».
A Johannesburg, les ambitions affichées par la municipalité de faire de la ville une «world-class city» passent par une telle logique de revitalisation économique du CBD et une volonté d'y attirer un nombre grandissant de capitaux, via un retour des entreprises et des consommateurs dans le centre-ville (GREATER JOHANnesburg Metropolitan Council (GJMC) 2003). Et, comme le mentionne un document publicitaire de la compagnie de vidéosurveillance,

«the Cueincident surveillance system is participating in maintaining Joburg's status as a world-class African city»

CUEINCIDENT 2003).

La vidéosurveillance est envisagée, par ses promoteurs, comme un moyen de faire disparaître la culture de la peur, tout en mettant en avant une nouvelle culture publique basée sur la consommation, à même de revitaliser le centre-ville.

Ces considérations permettent, en dernier lieu, de suggérer de façon plus précise les groupes sociaux qui, selon toute vraisemblance, sont les exclus du nouveau processus de territorialisation à l'œuvre dans le CBD. Comme le résument Bannister, Fyfe \& KeARns (1998:32)

«commercial imperatives define acceptable behaviour, excluding those who detract from the consumption experience».

Sur son site web, BAC répertorie une liste de défis auxquels l'entreprise doit faire face. Parmi ceux-ci figure «the increasing numbers of homeless and unemployed people living in urban centre streets» (BusIness Against Crime South Africa (BAC) 2003). De plus, un sondage effectué par BACST auprès d'acteurs de l'économie formelle du CBD mentionne parmi les «soucis» de ces derniers, à part la criminalité, le manque de contrôle des vendeurs de rues, les foules de sans-emplois et les activités anti-sociales inacceptables, ainsi que la désintégration de la vie sociale (Fraser 2003). En définitive, le constat qui se dégage de ce genre d'évolutions est proche de celui de FyFE \& Bannister (1996: 43), lesquels, en s'appuyant sur Mulgan (1989), stipulent que

«attempts to create a «convivial milieu〉 for economic and socio-cultural life in the city using CCTV may become attempts to purify space of those <troublesome others > - the underclass, the homeless, the unemployed».

\section{Conclusion}

Dans cet article, nous avons suggéré que la vidéosurveillance dans le CBD de Johannesburg, à travers un processus de territorialisation, contribue à l'instauration de ce que ZUKIN (1995: 3) appelle une «culture publique privatisée» et non inclusive. En d'autres termes, comme le disent Bannister, Fyfe \& Kearns (1998:27), 
«the message carried within this form of urban management is clear: difference is not so much to be celebrated as segregated, difference carries less value than it does danger».

De cette façon, la vidéosurveillance, au lieu d'altérer la «culture de la peur», comme cela peut apparaître au premier abord, semble au contraire la renforcer, bien que désormais, dans le contexte post-apartheid, la peur semble être plus celle de l'autre socio-économiquement différent, que celle de l'autre «racialement» différent.

Il ne faut cependant pas concevoir la pratique de vidéosurveillance de façon isolée à l'intérieur de ce processus. Elle peut, en effet, être assimilée à ce que Foucault (2004:111) appelait des «dispositifs de sécurité»-qui ont pour cible principale la population -, et comprise dans un ensemble de «nouvelles techniques d'exclusion» qui accompagnent une nouvelle régulation de l'espace, ou en terme foucaldien, une (nouvelle) «gouvernementalité spatiale» (Murray 2004 24-28). En dernier ressort, il devient légitime de se poser la question du projet de société post-apartheid qui est contenu dans ce processus de territorialisation et de se demander dans quelle mesure une nouvelle forme de ségrégation urbaine post-apartheid n'est pas en train de se mettre en place en Afrique du Sud. Cette dernière prendrait appui, cette fois-ci, sur un syndrome sécuritaire, qui aurait succédé au «syndrome sanitaire» à l'origine de la ville d'apartheid (Guillaume 2001: 8689).

\section{Bibliographie}

Bannister, J., Fyfe, N. \& A. Kearns (1998): Closed circuit television and the city. - In: Norris, C., Moran, J. \& G. Armstrong (éds): Surveillance, closed circuit television and social control. - Ashgate: Aldershot. Bétin, C., Martinais, E.\& M.-C. Renard (2003) : Sécurité, vidéosurveillance et construction de la déviance: l'exemple du centre-ville de Lyon. - In: Déviance et société 27, 1:3-24.

Bremner, L. (1998): Crime and the emerging landscape of post-apartheid Johannesburg. - In: JUDIN, H. \& I. VLADISLAVIC (éds): Blank architecture, apartheid and after. - Rotterdam: NAI Publishers.

Business Against Crime South Africa (BAC) (2003): Web site http:/www.bac.co.za 14.06.03.

Christopherson, S. (1994): The fortress city: privatized spaces, consumer citizenship. - In: AмпN, A. (éd.): Postfordism: a reader. - Oxford: Blackwell.

Ciaran, R. (2003): Eternal vigilance is the price of liberty. - In: Leadership, October.

Cox, A. (2003): Cameras in Joburg cut crime by $80 \%$. - In: Saturday Star. - http://www.iol.co.za 7.2.2003.

CueIncident (2003): Watching over you in Joburg.
- Johannesburg: Cueincident Surveillance Technology. Cueincident (2004): Web site http://www.cueincident. com 07.07.04.

Cueincident Surveillance Technology, JohanNeSburg, Marketing and Communication Division (2004): Communication personnelle; 24 mars 2004.

Cueincident Surveillance Technology, Johannesburg, Operations Projects Division (2004): Communication personnelle; 24 mars 2004.

Dick, P.K. (2002 [1956]): Minority report. - Londres: Gollancz.

DirsuweIt, T. (2002): Johannesburg: fearful city? - In Urban forum 13, 3: 3-19.

Foucault, M. (2004): Sécurité, territoire, population. Cours au Collège de France, 1977-1978. - Paris: Gallimard et Seuil.

Fraser, N. (2003): Celebrating positive changes. - In: Citichat. - http://www.joburg.gov.za/citichat/2003/ june20_citichat.stm 20.06.03.

Fyfe, N. \& J. Bannister (1996): City watching: closed circuit television surveillance in public spaces. - In: Area 28, 1:37-46.

Fyfe, N. \& J. Bannister (1998): «The eyes upon the street», closed-circuit television surveillance and the city. - In: Fyfe, N. (éd.): Images of the street, planning, identity and control in public spaces. - Londres et New York: Routledge.

Greater Johannesburg Metropolitan Council (GJMC) (2003): Business guide. - http://www.joburg. co.za 30.09.03.

Greater Johannesburg Metropolitan Council (GJMC) (2004): Avoiding crime. - http://www.joburg. co.za 07.01.04.

Guillaume, P. (1997): Du blanc au noir... Essai sur une nouvelle ségrégation dans le centre de Johannesburg. - In: L'Espace géographique 1:21-33.

Guillaume, P. (2001): Johannesburg, géographies de l'exclusion. - Paris et Johannesburg: Karthala/Institut Français d'Afrique du Sud (IFAS).

Guillaume, P. (2004): La violence urbaine à Johannesburg. Entre réalité et prétexte. - In: Geographica Helvetica 59, 3: 188-198.

Institute for Security Studies (ISS), Pretoria, Crime and Justice Program (2004): Communication personnelle; 11 mars 2004.

LÉvy, J. (1994): L'espace légitime. Sur la dimension géographique de la fonction politique. - Paris: Presses de la Fondation Nationale des Sciences Politiques.

Lyon, D. (2001): Surveillance society, monitoring everyday life. - Buckingham et Philadelphie:The Open University Press.

Mulgan , G. (1989): A tale of new cities. - In: Marxism today, March: $18-25$.

Murray, M. (2004): The evolving spatial form of cities in a globalising world economy, Johannesburg and São Paulo. - Cape Town: HSRC Publishers.

Norris, C. \& G. Armstrong (1998): Introduction: 
power and vision. - In: Norris, C., Moran, J. \& G. Armstrong (éds): Surveillance, closed circuit television and social control. - Ashgate: Aldershot.

November, V., KLauser, F. \& J. Ruegg (2002): Risques sous surveillance: une analyse géographique de l'utilisation de la vidéosurveillance. - In: Ethique publique 4, 2: 153-164.

Plessis, M. DU \& B. Holtmann (2002): An assessment of electronic surveillance in Gauteng. - Pretoria: Council for Scientific and Industrial Research (CSIR), Crime Prevention National Research Resources Centre.

RAFFEstin, C. (1997): Le rôle des sciences et des techniques dans les processus de territorialisation. - In: Revue européenne des sciences sociales 35, 108: 93 106.

South African Police Service (SAPS) (2003): Annual report of the national commissioner of the SAPS. 2002/2003 Financial year. - Pretoria.

South African Police Service (SAPS), Johannesburg Central Police Station, Crime Prevention Unit (2004): Communication personnelle; 7 avril 2004.

Tomlinson, R. (1999): From exclusion to inclusion. Rethinking Johannesburg's central city. - In: Environment and planning A 31: 1655-1678.

VITALis, A. (1998): Etre vu sans jamais voir. Le regard omniprésent de la vidéosurveillance. - In: Le Monde diplomatique, mars: 26-27.

Zukin, S. (1995): The cultures of cities. - Cambridge et Oxford: Blackwell.

\section{Résumé: Vidéosurveillance à Johannesburg. Impacts sur la population et le territoire}

Afin de combattre la criminalité et la violence, Johannesburg, dont l'ambition est de devenir une «worldclass city», s'est dotée d'un système de vidéosurveillance dans son Central Business District (CBD). Cet article analyse les impacts sociaux et spatiaux de cette nouvelle technique de surveillance. Il est argumenté que le système de caméras vise principalement une défense des lieux de pouvoir, et que ses effets sur la criminalité sont incertains. Par ailleurs, la vidéosurveillance contribue à une nouvelle territorialisation de l'espace post-apartheid et à l'instauration d'une culture publique privatisée et non inclusive, qui semble exclure toute une frange de la population en fonction de ses caractéristiques socio-économiques.

\section{Summary: Closed circuit television (CCTV) in Johan-} nesburg. Effects on residents and the surrounding area In order to combat violence and criminality, Johannesburg, in its striving to become a «world-class city», has installed a closed circuit television system in its Central Business District (CBD). The article analyses the social and spatial effects of this modern surveillance technology. It is argued that surveillance by camera essentially aims at protecting important areas. Its effect on reducing criminality does not appear to be as certain. Furthermore, closed circuit television has contributed towards a new territorialisation of space in the wake of Apartheid and towards the introduction of a privatized public culture. This means that a whole segment of the population appears to be excluded from such areas on socio-economic grounds.

\section{Zusammenfassung: Videoüberwachung in Johannes- burg. Auswirkungen auf die Bevölkerung und den Raum}

Um die Gewalt und Kriminalität zu bekämpfen, hat Johannesburg, das eine «world-class city» werden möchte, ein Videoüberwachungssystem in seinem Central Business District (CBD) eingerichtet. Der vorliegende Beitrag analysiert die sozialen und räumlichen Auswirkungen dieser modernen Überwachungstechnik. Es wird argumentiert, dass die Überwachung mit Kameras hauptsächlich darauf zielt, wichtige Orte zu schützen, dass der Einfluss auf die Kriminalität aber unsicher ist. Ausserdem bewirkt die Videoüberwachung eine neue Territorialisierung des Raumes nach der Apartheid und die Einführung einer privatisierten und nicht einschliesslichen öffentlichen Kultur. Dies bedeutet, dass ein ganzes Bevölkerungssegment aufgrund seiner sozioökonomischen Charakteristika ausgeschlossen wird.

Lic. géo. Lucas Oesch, assistant diplômé, Institut Universitaire d'Etudes du Développement (IUED), 20 rue Rothschild, CP 136, CH-1211 Genève 21, Suisse. e-mail: Lucas.Oesch@iued.unige.ch

\section{Manuskripteingang/received/manuscrit entré le 21.3.2006}

Annahme zum Druck/accepted for publication/accepté pour limpression: 13.6.2007 